\title{
De résoudre un problème à problématiser mathématiquement
}

Vers une nouvelle approche de l'activité mathématique de l'élève

\section{From problem solving to problematizing mathematically}

\author{
A new approach to student mathematical activity
}

\section{De la resolución de problemas a problematizar} matemáticamente

\section{Hacia un nuevo enfoque de la actividad matemática del alumno}

\section{Jean-François Maheux et Jérôme Proulx}

Volume 42, numéro 2, automne 2014

Résolution de problèmes en mathématiques : un outil pour enseigner et un objet d'apprentissage

URI : https://id.erudit.org/iderudit/1027904ar

DOI : https://doi.org/10.7202/1027904ar

Aller au sommaire du numéro

\section{Éditeur(s)}

Association canadienne d'éducation de langue française

ISSN

1916-8659 (numérique)

Découvrir la revue

Citer cet article

Maheux, J.-F. \& Proulx, J. (2014). De résoudre un problème à problématiser mathématiquement : vers une nouvelle approche de l'activité mathématique de l'élève. Éducation et francophonie, 42(2), 24-43.

https://doi.org/10.7202/1027904ar
Résumé de l'article

Dans cet article, nous proposons de considérer l'activité mathématique de l'élève qui traite un " problème " en remplaçant l'idée de " résolution de problème » par celle de problématisation mathématique. Cette reformulation correspond en fait à un changement de perspective sur le plan épistémologique; elle cherche à mettre en évidence l'idée que l'élève, face à une tâche, répond nécessairement au problème qu'il se pose lui-même. Nous illustrons ce changement paradigmatique à l'aide d'exemples divers et nous montrons comment l'activité de problématiser mathématiquement est elle-même en constant mouvement, révélant toute l'importance d'une perspective considérant le point de vue de l'observateur (que ce soit l'élève, l'enseignant ou un chercheur, par exemple). Prenant en partie sa source dans la théorie de l'enaction, cette proposition conduit à porter un regard sur l'activité mathématique fondée sur le faire mathématique (voir Maheux et Proulx, 2014), plutôt qu'en référence à ce qui serait " mathématiquement bon " dans une sorte d’absolu désincarné.
Tous droits réservés (c) Association canadienne d'éducation de langue française, 2014 services d’Érudit (y compris la reproduction) est assujettie à sa politique d'utilisation que vous pouvez consulter en ligne.

https://apropos.erudit.org/fr/usagers/politique-dutilisation/ 


\section{De résoudre un problème à problématiser mathématiquement Vers une nouvelle approche de l'activité mathématique de l'élève}

Jean-François MAHEUX

Université du Québec à Montréal, Québec, Canada

Jérôme PROULX

Université du Québec à Montréal, Québec, Canada

\section{RÉSUMÉ}

Dans cet article, nous proposons de considérer l'activité mathématique de l'élève qui traite un "problème» en remplaçant l'idée de «résolution de problème» par celle de problématisation mathématique. Cette reformulation correspond en fait à un changement de perspective sur le plan épistémologique; elle cherche à mettre en évidence l'idée que l'élève, face à une tâche, répond nécessairement au problème qu'il se pose lui-même. Nous illustrons ce changement paradigmatique à l'aide d'exemples divers et nous montrons comment l'activité de problématiser mathématiquement est elle-même en constant mouvement, révélant toute l'importance d'une perspective considérant le point de vue de l'observateur (que ce soit l'élève, l'enseignant ou un chercheur, par exemple). Prenant en partie sa source dans la théorie de 
l'enaction, cette proposition conduit à porter un regard sur l'activité mathématique fondée sur le faire mathématique (voir Maheux et Proulx, 2014), plutôt qu'en référence à ce qui serait «mathématiquement bon» dans une sorte d'absolu désincarné.

\section{ABSTRACT}

\section{From problem solving to problematizing mathematically - a new approach to student mathematical activity}

Jean-François MAHEUX

University of Quebec in Montreal, Quebec, Canada

Jérôme PROULX

University of Quebec in Montréal, Quebec, Canada

In this paper, we approach the student mathematics activity that addresses a "problem" by replacing the idea of "problem solving" by that of problematizing mathematically. This reformulation corresponds to a change in perspective on the epistemological level - it seeks to highlight the idea that faced with a task, the student answers the question he asks himself. We illustrate this paradigm shift with various examples and show how the activity of problematizing mathematically is itself in constant movement, revealing all the importance of a perspective that considers the point of view of the observer (whether student, teacher or researcher, for example). Taking its source in part from the theory of enaction, this proposal leads us to take a look at the mathematical activity based on doing mathematics (see Maheux \& Proulx, 2014), rather than in reference to what would be "mathematically sound" in a kind of disembodied absolute.

\section{RESUMEN}

De la resolución de problemas a problematizar matemáticamente: hacia un nuevo enfoque de la actividad matemática del alumno

Jean-François MAHEUX

Universidad de Quebec en Montreal, Quebec, Canadá

Jérôme PROULX

Universidad de Quebec en Montreal, Quebec, Canadá

En este artículo proponemos un enfoque de la actividad matemática del alumno que trata un «problema» remplazando la idea de «resolución de problema» por problematizar matemáticamente. Esta reformulación corresponde de hecho a un 
cambio de perspectiva en el plano epistemológico; trata de evidenciar la idea de que el alumno, frente a un trabajo, necesariamente responde al problema que él mismo se ha puesto. Ilustramos este cambio paradigmático con la ayuda de ejemplos diversos y mostramos como la actividad de problematizar matemáticamente está, ella misma, en constante movimiento, lo que revela la importancia de una perspectiva que tome en consideración el punto de vista del observador (sea el alumno o el investigador, por ejemplo). Enraizándose parcialmente en la teoría de la enacción, esta proposición nos lleva a ver la actividad matemática basada en el hacer matemático (ver Maheux \& Proulx 2014) en vez de en referencia a lo que sería «matemáticamente bueno», como algo de absoluto desencarnado.

La problématisation suppose d'identifier les traits significatifs ou encore "relevants" par rapport au contexte de l'interrogation

M. Fabre (2009, p. 226)

\section{Introduction}

Par des travaux souvent réunis sous le vocable «résolution de problème», la recherche en didactique des mathématiques s'intéresse depuis de nombreuses années à la manière dont les élèves (et les enseignants) abordent mathématiquement diverses situations. Ainsi, on définit volontiers un «problème» en parlant de l'élève qui «ne dispose pas» des procédures requises pour conclure sur un énoncé (voir le Fascicule K, Gouvernement du Québec, 1988). L'idée, qui occupe une place importante dans les programmes québécois depuis (au moins) les années 1980, a cependant évolué considérablement. En particulier, on reconnait de plus en plus que l'on ne peut fixer dans l'absolu le caractère problématique d'une situation, celui-ci étant toujours relatif à celui ou celle qui la traite. Témoin de cette évolution, ce qui fut un temps la recherche de «situations fondamentales» en didactique des mathématiques (p. ex. Brousseau, 1988), c'est-à-dire de problèmes que seule l'invention de concepts précis (déterminés par l'enseignant comme objets d'apprentissage) permet de résoudre de façon optimale, élargit aujourd'hui son spectre pour parler de situations «significatives» (situations qui permettent de justifier l'ensemble des stratégies et des concepts visés) ou de situations «initiales d'un processus génétique», c'est-à-dire faisant partie d'une chaîne de situations visant à aborder un concept (Brousseau, 2005). On peut aussi, par exemple, penser aux travaux de Rogalski (2003) qui, depuis une dizaine d'années, conceptualise les multiples réinventions d'une tâche donnée par l'enseignant et les élèves eux-mêmes. Mais l'idée selon laquelle la situation dépend de manière essentielle de la personne qui la rencontre reste cependant assez 
périphérique: cette nuance, traditionnellement prise en compte pour définir les limites d'une approche de l'activité de l'élève ou du travail de l'enseignant, semble rarement considérée comme suffisante pour remettre en question l'idée même de «résolution de problème». En fait, ces observations ne semblent pas avoir conduit à une réorientation majeure de la recherche autour de la résolution de problème, où ce sont surtout les caractéristiques mathématiques des situations (telles qu'interprétées par celui ou celle qui les propose) qui servent de base à l'étude de l'activité mathématique (Xin, 2012; Armstrong, 2013).

Un autre exemple allant dans le même sens concerne les "processus de résolutions» mis en œuvre lors du traitement d'un problème. Le fameux modèle "par étapes» inspiré de Polya (1965), qui suppose que la résolution d'un problème peut être divisée en une suite d'étapes bien définies (compréhension du problème, élaboration d'un plan, exécution du plan, obtention puis vérification de la solution), a peu à peu fait place à des approches plus ouvertes inspirées entre autres des travaux sur la modélisation (Bloom et Leiss, 2005). On donne alors une plus grande part à différentes heuristiques (simplifier, exemplifier, généraliser, etc.) conduisant à une appropriation du "problème» (p. ex. d'un énoncé). Radford (1996), par exemple, note que:

La compréhension [de l'énoncé d'un problème] est conditionnée entre autres par les connaissances antérieures de l'individu. De plus, le fonctionnement de la compréhension dépend du niveau de formation cognitive du modèle de résolution de problèmes auquel l'individu fera appel. [...] Il faut donc être conscients qu'en général on ne peut pas demander à l'élève d'avancer un plan, suite à la lecture du problème. La compréhension et l'élaboration d'un plan ne sont pas des «étapes» indépendantes; ce sont des processus interreliés. Probablement qu'il serait plus fructueux de demander à l'élève d'essayer de plonger dans le problème, d'essayer des solutions possibles (p. 28-29).

Ce qui l'amène par la suite à faire cette proposition:

En décodant le problème avec ses propres moyens, [l'élève] n'a pas compris la même chose [que nous]. Quoi de plus naturel alors que de [chercher à] voir le problème depuis sa propre perspective, c'est-à-dire depuis ses propres modèles de résolution de problèmes? (p. 30)

Cette proposition de Radford n'est pas anodine, car elle remet en question non seulement l'idée d'un problème qui existerait en dehors de l'élève, mais aussi l'image selon laquelle l'élève résout "un " problème dont la forme ou la nature sont fixées au départ. Cette suggestion dans le sens d'une coévolution du problème et de sa résolution ne semble cependant pas, elle non plus, avoir bousculé de manière importante les travaux sur la résolution du problème. Or, qu'en est-il si nous prenons au sérieux la proposition selon laquelle un problème dépend rigoureusement de celui ou celle qui l'aborde, et de son travail mathématique sur celui-ci au fur et à mesure qu'il ou elle progresse dans sa «résolution»? 
Dans cet article, nous proposons d'aborder cette question, qui nous conduit à mettre de côté l'idée même de «résolution de problème», pour parler plutôt de l'activité qui consiste à problématiser mathématiquement. Pour ce faire, nous enracinons cette proposition dans une approche biologique de l'activité humaine et de la cognition à laquelle la recherche autour de l'éducation mathématique s'intéresse depuis de nombreuses années (Davis, Sumara et Kieren, 1996) et qui occupe aujourd'hui encore un bon nombre de chercheurs (voir par exemple le forum de recherche dans Proulx, Simmt et Towers, 2009, et le numéro spécial de ZDM dirigé par Reid, Brown, Coles et Lozano, à paraître). Dans la section suivante, nous rappelons brièvement quelques fondements au cœur de l'enaction. Nous nous y appuyons afin de parler de l'activité de problématiser mathématiquement, offrant par la suite quelques liens avec les travaux en "problem posing» qui intéressent la recherche en didactique des mathématiques depuis une vingtaine d'années (voir par exemple Silver, 2013).

\section{Une entrée sur l'activité mathématique par l'enaction}

Les travaux de Maturana et Varela (Maturana, 1988; Maturana et Varela, 1992, 1994; Varela, 1999; Varela, Thompson et Rosch, 1991), souvent associés au terme enaction, adoptent un point de vue biologique sur l'activité humaine et la cognition. La perspective biologique a souvent été utilisée en tant que métaphore pour penser la connaissance et l'apprentissage (voir notamment Piaget, Glasersfeld, Siegler, Morss, Dewey), par exemple avec l'idée d'adaptation, d'évolution, etc. Toutefois, pour Maturana et Varela, la cognition est littéralement un phénomène biologique, ce qui conduit à parler non plus de "connaissances", mais plutôt d'action adéquate, c'est-à-dire permettant de maintenir une relation (fonctionnelle) entre un organisme et son environnement. Ainsi, qu'il s'agisse d'une araignée tissant sa toile, d'une plante orientant ses tiges vers le soleil ou d'un élève répondant à une question mathématique, tous réalisent un acte qui leur permet de continuer à évoluer avec leur environnement, qui est lui-même en évolution. On dit alors de l'activité mathématique de l'élève (par exemple ce qu'on identifie comme ses interprétations, ses stratégies, ses solutions mathématiques) qu'elle est fonctionnelle par rapport au contexte (mathématique) dans lequel celui-ci évolue (Reid, 1996; Zack et Reid, 2003, 2004), contexte dont l'évolution est en partie provoquée par l'action même de l'élève.

Le concept d'action adéquate et son caractère fonctionnel tournent ainsi notre intérêt vers les manières dont les élèves travaillent mathématiquement, ayant soin de montrer comment ce travail est le fait d'interactions entre élèves, d'une part, et tâche ou contexte, d'autre part:

Enactivism prompts us to attend as closely to the preceding actions - the unformulated exploration, the undirected movement, the unstructured interaction, wherein the body is wholly engaged in mathematical play - as to the formal mathematical ideas that might emerge from those actions (Davis, Sumara et Kieren, 1996, p. 156). 
Il s'ensuit que l'activité mathématique, bien que liée à l'apprenant et à la tâche (Davis, 1995), ne peut être réduite ni à l'un ni à l'autre: elle émerge, toujours en mouvement, à la rencontre des deux (Davis et al., 1996). On dira par exemple que l'apprenant qui réalise une tâche mathématique fait émerger une stratégie située dans la tâche (Thom et al., 2009), la tâche ayant de son côté permis à cette stratégie de voir le jour. C'est la raison pour laquelle, comme l'explique René de Cotret (1999), on ne peut tenir pour acquis que des propriétés instructionnelles sont présentes de façon inhérente dans les situations offertes aux élèves; nous disons plutôt qu'elles émergent dans l'interaction ${ }^{1}$.

Parler d'action adéquate met de l'avant un autre point important de la perspective de Maturana, soit l'observateur: "Knowledge is adequate action in a domain specified by a questionner» (Maturana, dans Simon, 1985, p. 37). On attache ainsi l'aspect adéquat de l'action non pas à quelque référentiel objectif, mais au regard d'un observateur qui juge celle-ci. En particulier, la nature «mathématique» des tâches, de l'environnement ou de l'activité de l'élève (y compris, donc, sa qualité d'être «mathématiquement correcte») dépend de cet observateur (qui peut être l'élève lui-même ou son enseignant ou, encore, un chercheur):

Thus, if someone claims to know algebra - that is, to be an algebraist - we demand of him or her to perform in the domain of what we consider algebra to be, and if according to us she or he performs adequately in that domain, we accept the claim (Maturana, 1988, p. 4-5).

Si, d'une part, l'enjeu se porte alors sur la manière dont nous allons accepter ou non comme «mathématique» telle proposition d'un élève, il est important de remarquer que, d'autre part, l'action d'observer n'est pas prise en soi, mais abordée comme acte de distinction, de propos, de lecture, qui détermine ainsi l'objet de l'observation... de la même manière que l'action (mathématique) de l'élève! Les travaux autour de l'enaction attirent notre attention sur ce qui se réalise dans/avec une situation particulière pour y apprécier les mathématiques en jeu. Ces travaux offrent la possibilité de conceptualiser l'idée selon laquelle ce qui est proposé à un élève devient un problème, à condition d'être abordé comme tel, et continue d'évoluer sous son action mathématique. Un phénomène de circularité, souvent décrit par Maturana et Varela, est alors à l'œuvre: ce qui fait d'un problème un problème est d'être abordé comme tel, ce problème appelant en même temps les actions particulières... qui en font un problème. Or, qui dit circularité ne dit pas «tourner en rond»: c'est plutôt l'image d'une boucle itérative qu'il faut voir, ce que Davis et Simmt (2004) appellent une «élaboration récursive» et dont le mouvement produit ce que Kieren (1995) appelle «a world of mathematical significance» (p. 7) en constante évolution.

Nous abordons maintenant de manière plus spécifique l'idée du passage de «résoudre un problème» vers celle de problématiser mathématiquement à travers cette perspective théorique.

1. On peut relier cela au concept d'affordances de Gibson, comme nous l'avons fait dans Proulx (2014). 


\section{Problématiser mathématiquement}

Varela (1996; Varela et al., 1991) note que la notion de «résolution de problèmes» sous-entend que des problèmes existent en dehors de nous, déjà présents et en attente d'être résolus. Une telle indépendance par rapport à celui ou celle qui agit correspond fort mal aux observations effectuées dans le cadre des travaux en enaction mentionnés plus haut. Varela propose de considérer que nous spécifions les «problèmes» que nous rencontrons, à travers le sens que nous donnons au monde, et que nous sommes ceux qui voient et créent des problèmes à résoudre: bref, que nous posons des problèmes, que nous posons nos propres problèmes. Le verbe "problématiser» permet de mieux rendre compte de cette idée, insistant sur le fait que, du point de vue de l'enaction, il n'y a jamais véritablement quelque chose de "posé» ayant une signification fixe (un problème établi à résoudre), mais toujours une action produisant cette signification. Ce qu'on appelle un problème est alors la reconnaissance, par un observateur, d'une certaine régularité dans un processus suivi de problématisation. On conceptualise peut-être plus facilement cette idée en pensant à la distinction souvent faite entre «solution" et "résolution", la chose et le processus. Dans cette perspective, résoudre et problématiser sont une seule et même chose. La démarche de résolution et le problème résolu, tous deux observés a posteriori, se définissent l'un par rapport à l'autre (j'ai résolu le problème auquel ma solution répond!). Mais il s'agit aussi d'un processus vivant, fluide et hautement créatif qui se développe tout au long du travail mathématique. On peut par exemple penser à ce qui s'est produit lorsque nous avons demandé à des enseignants de mathématiques de déterminer mentalement l'aire du carré suivant:

Figure 1. Quelle est l'aire du carré?

Les nombreuses propositions que nous avons obtenues correspondent souvent à des manières fort différentes de "voir» la figure et de la "manipuler» mathématiquement. Ainsi, certains ont tout de suite proposé un traitement numérique, donnant aux carrés pointillés une mesure de 1 x 1 , pour utiliser ensuite la relation de 
Pythagore et trouver la mesure de leur diagonale (p. ex. $A B=\sqrt{ } 2$ ), puis celle du carré $A B C D$ par la formule d'aire $\left(\sqrt{ } 2^{\wedge} 2=2\right)$. D'autres, plus nombreux, ont procédé à différents découpages, se référant ou non au carré défini par les 4 points en périphérie dont la surface est clairement de " 4 unités», si l'on reprend le quadrillage comme étalon de mesure. Une participante nous a aussi expliqué qu'il n'y avait "pas de problème» là pour elle, car elle a immédiatement reconnu la figure du célèbre Ménon de Platon, dans lequel Socrate aide un esclave à doubler la surface d'un carré à l'aide de sa diagonale. L'idée ici est que, d'une part, ces propositions correspondent bel et bien à des problématisations différentes à partir de ce qui a été présenté: tantôt en termes numériques, alors traité par formules, tantôt en termes géométriques, faisant alors souvent appel à des isométries formulées de manière plus ou moins formelle. Mais, plus encore, nous nous avançons vers l'idée selon laquelle chaque «pas» dans la résolution, chaque observation, chaque manipulation correspond à l'émergence d'un nouveau problème. Par exemple, un retour sur une des stratégies par découpage décline une première étape, qui revient en fait à la formulation d'un nouveau problème: si l'on considère un déplacement du triangle $\mathrm{BCD}$ de manière à former un parallélogramme en l'appuyant sur le triangle ABD, quelle est maintenant l'aire de ce parallélogramme? La problématisation mathématique se fait tout au long du travail (et, pourrait-on dire, de tout travail mathématique!), et la résolution s'arrête avec celle-ci, que l'on ait ou non produit quelque chose de «satisfaisant» pour soi-même ou pour un autre...

Du point de vue de l'enaction, c'est ainsi avant tout dans les rencontres entre une personne et son environnement que se "posent» les problèmes, ou plutôt qu'ils se créent et se recréent, chacun faisant (chaque fois!) ces rencontres de manière personnelle et unique. On peut ainsi reconnaître l'idée de problématiser mathématiquement face à un énoncé aussi simple que « $2+5=$ ?». Chacun d'entre nous fait une expérience unique de cet énoncé et le problématise à sa manière. Cela ne veut pas dire qu'une fois interrogé sur la manière utilisée pour trouver un résultat chacun aura forcément une approche (mathématiquement) différente: au contraire, il y a fort à parier que la majorité d'entre nous reconnaîtront avoir recours aux mêmes "stratégies» et «idées», diront avoir fait une «addition», obtenu «7», etc. ${ }^{2 .}$ Toutefois, même si tous semblent pointer dans la même direction, et parler du même « 7 » en tant que résultat, chacun aura fait sa propre expérience des nombres présentés, de l'addition et du « 7 ». Chacun problématise l'énoncé « $2+5=$ ?» à sa manière, avec peut-être une façon particulière de concevoir l'addition. On ne pense pas uniquement aux «sens" les plus classiques associés à cette opération (réunion, déplacement, relation entre nombres), mais surtout à l'expérience personnelle de chacun, qui en fera chaque fois un exercice unique, avec des résonances singulières. Ainsi, personne ne «voit» ou ne «fait» l'addition exactement de la même manière, et même les nombres évoquent quelque chose d'incomparable pour chacun d'entre nous. Pour certains, le 7 est relié à des objets ou une collection, pour d'autres à un chiffre chanceux, pour d'autres à

2. Et là encore... Une collègue mathématicienne à qui nous avons présenté l'énoncé a réagi en nous demandant immédiatement de spécifier l'ensemble de nombres dans lequel l'opération est posée. En effet, on peut aussi avoir comme solution « $2+5=1$ (modulo 6)», et ainsi de suite! 
un célèbre défenseur des Oilers d'Edmonton, pour d'autres à une forme géométrique, pour d'autres à la $3^{\mathrm{e}}$ position avant 10 . D'autres, encore, peuvent surtout y retrouver un mauvais souvenir de mathématiques scolaires... Pour un observateur, la solution peut bien sembler "la même», avoir été obtenue par une série de démarches et de stratégies semblables correspondant à un énoncé «compris » de façon équivalente par tous. Chacun aura néanmoins rencontré, et donc problématisé mathématiquement, l'énoncé « $2+5=$ ?» de manière unique.

Une telle perspective sur le phénomène est intéressante pour aborder de manière constructive une foule d'observations, dont les résultats au fameux problème de «l'âge du capitaine» ne sont qu'un exemple (c'est-à-dire à un énoncé du type «Un bateau transporte 12 moutons et 15 chèvres, quel est l'âge du capitaine?» auquel plusieurs enfants répondent « 27 ans»). On voit bien qu'il s'agit de problématiser à partir de l'énoncé proposé, mais celui-ci n'étant pas porteur d'un problème (p. ex. absurde) de manière inhérente. L'énoncé porte au mieux un potentiel de problématisation se réalisant de manière variée selon l'élève, les circonstances, etc. Maturana (1987) aime à cet égard raconter l'histoire de l'étudiant à qui l'on demande de déterminer la hauteur d'un édifice à l'aide d'un baromètre et qui propose une pléiade de solutions "valables" (y compris celle d'échanger le baromètre contre l'information recherchée!), mais allant a contrario de celle typiquement attendue dans le cadre d'un examen de physique. Tournée vers l'enaction, l'idée de problématiser mathématiquement nous offre ainsi une voie médiane entre le réalisme représentationaliste (où un problème existerait en soi) et le solipsisme philosophique (que l'on reproche souvent au constructivisme radical, par exemple, et qui semble rendre théoriquement impossible/intenable la conceptualisation de quoi que ce soit «en dehors» de l'expérience individuelle) (voir Maturana et Varela, 1992; Varela, 1996). De façon plus fine, on considère aussi, par exemple, la manière dont les étudiants peuvent faire de la résolution d'équations algébriques, telles que « $6 / x=3 / 5$ », un problème de multiplication, de ratio, de fonction, d'algèbre, etc. (voir Proulx, 2013a). Là encore, on a une série de problématisations qui dépendent à la fois de ce qui est présenté aux étudiants (p. ex. l'énoncé « $6 / x=3 / 5$ » écrit au tableau) et de la lecture qu'ils en font, elle-même sans doute enracinée dans des manières de faire reconnaissables sur le plan individuel ou collectif. On rencontre également cette idée, et de manière très articulée, chez Foucault (p. ex. 1985) lorsqu'il parle de problématisation:

Problematization doesn't mean the representation of a pre-existing object, nor the creation through discourse of an object that doesn't exist. It is the set of discursive and non-discursive practices that makes something enter into the play of the true and the false and constitutes it an object for thought. [...] (Foucault, 1988, cité dans Bacchi, 2012, p. 4).

Foucault is looking to carve out a space between realism and idealism. That is, he postulates that there is something "real" being regulated. Problematization is a description of thinking as a practice (Bacchi, 2012, p. 3). 
Ce détour par le philosophe, sociologue et historien français apparait pertinent à l'égard du traitement détaillé qu'il donne à l'idée de problématisation qui, à l'échelle de son œuvre, va jusqu'à prendre le statut de méthode. Pour Foucault, penser en termes de problématisation conduit à considérer comment et pourquoi certaines choses deviennent des problèmes, et comment ces «choses" sont (trans)formées en «objets» pour la pensée (voir, par exemple, l'analyse de Deacon, 2000). Ses écrits ne portent évidemment pas sur la problématisation mathématique qui nous intéresse ici. Ils concernent plutôt les phénomènes sociaux, tels que la folie, la délinquance ou le pouvoir, mais l'idée de s'intéresser aux conditions dans lesquelles un problème se crée, à sa généalogie possible, est porteuse pour aborder l'activité mathématique. Du point de vue de l'enaction, il n'y a d'ailleurs pas de rupture véritable entre ontogénie, évolution sociale et phylogénie (Maturana et Varela, 1994), le même type de processus étant à l'œuvre, un peu à la façon dont les cellules, les organes et le corps dans leur ensemble peuvent à tour de rôle être considérés comme des organismes en interaction avec leurs environnements (produisant ceux-ci tout en étant produits par eux). Ainsi, l'idée selon laquelle la problématisation est un processus historique dont les aléas permettent d'apprécier le caractère fondamentalement émergeant, relationnel et contextuel de ces «objets» dont on s'occupe, répond bien - à l'échelle de l'expérience mathématique - au principe selon lequel chacune de nos actions est le fruit de l'ensemble de notre histoire (Davis, Sumara et Kieren, 1996; Brown et Coles, $2012)^{3}$.

En fait, il existe toute une tradition de réflexion sur la notion de "problème» et sur l'activité de problématisation dont on peut tirer des leçons. On peut penser, par exemple, au travail de Meyer (1986), qui examine la question du point de vue de l'histoire de la philosophie. Meyer souligne comment on a longtemps travaillé dans le «non-penser problématologique», qu'il rattache à l'idée de "faits», au lieu de prendre pour base de travail le phénomène d'ouverture que provoque une attitude de questionnement (un problème pouvant toujours mener à une multitude d'autres problèmes $)^{4}$. Une illustration toute simple du changement d'orientation que cela suggère peut être trouvée du côté des fameux "faits mathématiques» qui peuvent donner l'impression de devoir être mémorisés, ayant peu à offrir en termes «conceptuels» (pour reprendre la distinction entre [compréhensions] mathématiques factuelles et conceptuelles souvent rencontrées dans les écrits scientifiques). Or, nous savons très bien que les tables d'addition ou de multiplication, par exemple, peuvent tout à fait être travaillées de façon conceptuelle, en termes de relations entre nombres, de régularités, voire par le biais du «calcul réfléchi». Comme avec l'addition discutée plus haut, on peut ainsi problématiser l'énoncé « $9 \times 7=$ × y voir une occasion de questionnement et donc d'engagement dans une activité mathématique variée, que ce soit pour penser à l'aide d'autres résultats et de propriétés $(9 \times 7=$ $10 \times 7$ - 1 x 7, etc.), ou de disposition géométrique, etc. Mayer valorise une aptitude à

3. Étymologiquement, les mots "objet» et "problème» sont d'ailleurs très proches, à travers deux formes du verbe «jeter »: jacere (pour ob-jet) et ballein (pour pro-blème).

4. On pourrait aussi se tourner vers Deleuze (1968), qui propose également une réflexion profonde autour de la notion de problème à travers I'histoire des sciences (dont Mayer parle également), ou vers Fabre (2009), cité en exergue, ou encore vers Bachelard (1949)... et vers bien d'autres encore! 
mettre en question plutôt qu'à "mettre en réponse» (p. 204) et nous invite ainsi à remettre la question en question (à problématiser la notion de problème), en particulier à l'égard des réponses qui... posent problème (p. ex. à propos de l'âge du capitaine, discuté précédemment)! Ce tournant vers une approche en termes d'attitude, de disposition, qui garde actif le phénomène de problématisation au lieu de l'enfermer dans une activité ou un moment particuliers, colle bien à l'esprit du travail où l'on encourage une forme de conscientisation (en anglais «awareness») permettant de considérer le caractère local, contingent, de ce qu'on identifie comme des questions, des réponses, des problèmes, des solutions, des erreurs, des connaissances. L'aspect récursif introduit par Meyer (problématiser, puis problématiser la problématisation, et problématiser cette problématisation, et ainsi de suite) est en phase avec plusieurs éléments de la démarche en enaction, dont l'idée de circularité mentionnée plus haut (p. ex. Maturana, 1988; Maturana et Varela, 1992; Varela, 1988, 1999). Les réponses qui posent problème nous ramènent alors au travail de l'observateur, qui est également une forme de problématisation. Poussant à l'extrême, on peut sans doute aller jusqu'à suggérer qu'enseigner les mathématiques n'est autre chose que de participer à la problématisation mathématique des élèves à partir de ce qu'on leur présente et qu'apprendre les mathématiques c'est peut-être essentiellement s'engager dans un «champ problématique» (expression empruntée à Deleuze, 1968, p. 214), où «faire mathématique» (Maheux et Proulx, 2014) permet de poursuivre l'enchaînement des questions et des réponses, c'est-à-dire la problématisation mathématique elle-même.

On dira sans doute que nous risquons fort, par une telle approche, de perdre en quelque sorte la spécificité du genre d'activités auxquelles on pense quand on évoque la «résolution de problème». En retour, cet élargissement de la notion peut nous amener à réfléchir davantage aux particularités des situations ou du travail que nous désignons généralement ainsi, et dont l'expression "résolution de problème" masque en quelque sorte la richesse et la diversité. Il s'agit également, et en toute cohérence, de penser que le chercheur en didactique des mathématiques est aussi occupé à problématiser mathématiquement, que ce soit au moment de concevoir des situations, d'analyser des productions d'élèves ou le travail d'enseignants, voire de communiquer à propos de ses observations!

\section{Et le "problem posing»?}

Avec cette proposition de reformulation de l'idée de résolution de problème vers celle de problématisation mathématique, il semble important et intéressant de faire un lien avec d'autres travaux en didactique des mathématiques. Plusieurs courants de recherche pourraient être mis à contribution, l'idée de problématisation telle qu'elle a été discutée plus haut n'étant pas sans rappeler le concept de mathématisation par exemple (Bauersfeld, 1994; Brousseau, 1998), voire de modélisation mathématique. Vu l'espace disponible ici et le thème de ce numéro spécial, nous nous contentons d'évoquer quelques pistes du côté de ce qu'on appelle en anglais le 
"problem posing» (qui se compare bien avec l'expression "problem solving»), et qui recouvre en gros ce qu'on envisage en le traduisant par l'expression composition de problèmes. Ce genre d'activité, rapporté depuis de nombreuses années (Polya, 1965; Silver, 1994; Brown et Walter, 2005; Kilpatrick, 1987; numéro thématique de ESM, 2013), offre plusieurs parallèles avec ce que nous avons décrit à propos de la problématisation mathématique, en plus d'être souvent discuté en lien avec ce qu'on appelle généralement la résolution de problème. On garde cependant en tête que, selon la perspective proposée ici, il n'est pas question de distinguer le traitement d'un énoncé (vu comme venu de l'extérieur) de la formulation d'un énoncé pouvant faire l'objet d'un traitement mathématique, l'idée fondamentale étant justement que ces deux types d'activités ne sont pas différents l'un de l'autre dans l'expérience qu'on en fait (voir par exemple nos travaux dans Proulx, 2013b; voir aussi Sevim et Cifarelli, 2013). Si s'engager dans la résolution d'un problème c'est forcément problématiser mathématiquement ce qui nous est proposé (qu'il s'agisse d'un énoncé ou d'un "problème sans mots" comme on en voit de plus en plus, par exemple dans Voica et al., 2013), il en va de même quand il s'agit de composer entre eux divers éléments afin de proposer une situation ou un énoncé pouvant être abordé mathématiquement.

En ce sens, bien que la problématisation mathématique déborde largement le cadre de la formulation de problèmes, plusieurs observations issues de la recherche sur ce type d'activité peuvent nous aider à articuler l'idée lancée par Varela dans le cadre des travaux autour de l'enaction. On pense par exemple aux propos de Kilpatrick (1987), qui souligne le caractère créatif de la reformulation de problèmes faisant émerger des liens, des idées. Prenant en compte l'idée selon laquelle celui ou celle qui aborde mathématiquement une situation doit la problématiser mathématiquement pour qu'elle existe à ce moment comme problème, un travail semblable de génération de liens et d'idées mathématiques est certainement à l'œuvre dans ce qu'on pourrait appeler la (re)formulation en tant que problème. Une nuance importante tient cependant au fait que ce processus de reformulation n'est pas nécessairement explicite même pour celui ou celle qui problématise. L'idée de rendre mathématiquement accessible un énoncé par (re)formulation (Whitin, 2004) est également intéressante: pour problématiser, on a recours a des idées mathématiques, ne seraitce que pour présager la pertinence du travail en cours (aspect fondamental de l'activité mathématique selon Hadamard (1959) par exemple). On reconnaît aussi dans la problématisation mathématique l'idée d'accepter ou de contester ce qui est «donné» par une situation («accepting the givens» et «challenging the givens») comme point de départ pour l'investigation mathématique de la situation rencontrée (Brown et Walter, 2005) (pensez à nouveau à l'âge du capitaine!). Toujours dans le même sens, on trouve même chez Duncker (1945), par exemple, une proposition selon laquelle toute avancée sur un problème est en fait une reformulation du problème en question. Ces (re)formulations «locales» s'appuient naturellement sur des définitions, des règles, bref, sur des idées mathématiques relativement familières (Hershkowitz, Schwarz et Dreyfus, 2001) qu'il s'agit de mettre "en discussion» avec la situation (Banwell, Saunders et Tahta, 1972). Et on reconnaît de plus en plus le caractère ouvert, non fini, de ce dialogue, chaque "problème» conduisant à de nouveaux problèmes 
dont l'ensemble pourra éventuellement être interprété comme une résolution de celui qui aura stimulé cette génération (Lewis, Petrina et Hill, 1998). Dans sa thèse, Armstong (2013) cite en ce sens les propos de Vera et Crossman (2004) concernant le travail de création de l'artiste:

As part of the creative process, actors find a problem for themselves, spend some time solving the problem, and find a new problem during the solving of the last one (p. 737).

D'une part, il s'agit évidemment d'un élargissement important du travail fondateur de Silver (1994), qui ouvrait déjà cette voie en reconnaissant au problem-posing une présence aux trois «moments» d'une investigation mathématique: avant d'avoir une solution, à l'intérieur de la résolution, et après ce processus. D'autre part, le rapprochement proposé par Armstrong avec les idées de Bauersfeld (1994) en termes de rafistolages et de bricolages («tinkering and bricolage»), caractéristiques du côté "confus» et des "adaptations pragmatiques» qu'il rattache à l'activité mathématique, nous ramène alors tout à fait aux propos de Varela et à sa distinction entre une approche en termes de "problèmes " qui existeraient en dehors du travail de celui ou celle qui les rencontres et la sérieuse prise en compte du travail de problématisation inhérent à toute approche (mathématique) d'une situation.

C'est une idée que l'on retrouve également du côté de la recherche sur la composition de problèmes mathématiques par des élèves, où il a été démontré que la formulation d'énoncés peut conduire à déclencher une activité mathématique productive et prolongée, mettant en oeuvre un processus continu de composition de problèmes subsidiaires (Christou, Mousoulides, Pittalis, Pitta-Pantazi et Sriraman, 2005; Ponte et Matos, 1992). Inspiré par l'enaction, nous insistons alors sur le fait que cet effet «déclencheur» ne répond pas à une logique de cause à effet, mais crée des occasions (Kieren, 1995, utilise en anglais le verbe "to occasion»), la résultante dépendant évidemment de celui ou celle à qui ces occasions s'offrent. Chacune de ces problématisations conduit à une activité mathématique différente, avec des buts et intentions différentes, mariant très étroitement ce qu'on pourrait appeler la composition et la résolution de problème (voir ici aussi nos propos dans Proulx, 2013b, et ceux de Sevim et Cifarelli, 2013).

Il va de soi que nous faisons ici une lecture fort orientée des écrits autour du problem-posing afin de mettre en lumière ce que nous présentons comme l'activité mathématique de l'élève inspirée par les écrits en enaction ${ }^{5}$. L'essentiel pour nous est d'aider à apprécier l'idée d'une perspective où les problèmes que nous rencontrons et les questions que nous posons font autant partie de nous que de notre environnement: ils émergent continuellement de notre constante interaction avec lui. Nous interprétons les évènements qui nous entourent comme des éléments à aborder, nous les vivons comme des problèmes à résoudre:

5. On pourrait évidemment faire aussi l'exercice autour de la recherche sur la résolution de problème ellemême, s'intéresser au travail mathématique, aux heuristiques qui y sont présentées sous l'angle particulier de la problématisation: nous souhaitons en fait inviter le lecteur à faire cet exercice, voire à (re)lire les articles composant ce numéro thématique sous cet angle... 
La plus importante faculté de toute cognition vivante est précisément, dans une large mesure, de poser les questions pertinentes qui surgissent à chaque moment de notre vie. Elles ne sont pas prédéfinies mais enactées, on les fait-émerger sur un arrière-plan, et les critères de pertinence sont dictés par notre sens commun, d'une manière toujours contextuelle (Varela, 1996, p. 91).

L'interaction en continu avec l'environnement crée en même temps les situations sur lesquelles nous agissons et nos manières d'agir sur elles. Bien sûr, il n'est pas question ici de détermination stricte et prédictible. On parle plus justement de l'ouverture vers un monde de possibles dont l'indétermination est à la fois ce qui permet qu'un "problème» existe et qu'une solution soit probable (mais non certaine): dialectique dont le fameux travail de Gödel a démontré être au cœur de toute entreprise mathématique. L'idée d'une rencontre aux conséquences inattendues entre un organisme et son environnement, aux racines du travail en enaction, conduit naturellement à considérer que si tels éléments de l'environnement déclenchent des réactions chez certaines personnes, ce ne sera pas nécessairement le cas chez d'autres, d'où l'idée qu'il n'y a pas de "problème» qui soit là "en attente», mais plutôt l'émergence de problème dans l'interaction avec, par exemple, une tâche. Dans les mots de Simmt (2000), on ne donne pas de problèmes aux élèves, mais plutôt des énoncés avec lesquels ils font des problèmes: l'énoncé devient problème quand l'élève en fait un problème.

\section{Problématiser mathématiquement - vers une nouvelle conceptualisation de l'activité mathématique de l'élève}

Dans ce qui précède, nous avons proposé de considérer ce qu'on appelle généralement la "résolution de problème» en tant que problématisation mathématique. Prenant en partie notre inspiration dans l'enaction, cette reformulation, qui nous amène à considérer le problème par l'élève (et non en dehors de lui ou elle), s'appuie sur une approche particulière de l'activité mathématique fondée sur l'idée que nous avons évoquée ailleurs de faire mathématique (Maheux et Proulx, 2014). Dans une telle approche du faire mathématique, l'attention se porte non pas sur le savoir ou sur les connaissances - qui sont comme les deux côtés d'une même lentille nous conduisant, comme observateurs, à «voir» l'activité mathématique en termes de «choses» (p. ex. connues ou à connaître) - mais sur l'activité mathématique elle-même, dont l'essentiel est peut-être de la rendre possible, voire désirable. Conceptualiser l'activité mathématique de l'élève en termes de problématiser mathématiquement nous pousse dans cette même direction.

Il s'ensuit que nos recherches, présentées ici surtout au niveau théorique avec quelques illustrations tirées de nos travaux en cours, n'ont pas pour objectif de mettre au point ou de tester ce qui pourrait être une ou des «méthodes» pour apprendre ou enseigner des mathématiques. Nous nous intéressons plutôt à développer de nouvelles 
façon d'aborder l'activité mathématique de manière à générer de nouvelles voies, de nouvelles questions pour la recherche, et peut-être, éventuellement, de nouvelles possibilités pour l'éducation mathématique (comme nous en parlons dans Proulx, 2010). Cela dit, le mot «méthode» pris au sens étymologique, du grec methodos qui signifie "chercher une voie, un chemin", n'est pas sans résonance avec la présente proposition (et on peut la ramener aux idées de Foucault citées plus haut). La nuance est sans doute que nous mettons l'accent sur la recherche elle-même, et son pouvoir générateur, plutôt que sur le chemin ou ce qui serait peut-être une «destination finale».

Penser que l'élève qui fait (des) mathématique(s) est engagé dans un processus où il problématise mathématiquement permet de formuler l'activité mathématique de la même manière, c'est-à-dire comme la recherche d'une voie, d'un chemin, dont le point d'arrivée nous est inconnu (sans quoi on ne parlerait pas de "problème» comme le dit Poirier, 2000) : le chemin se fait en marchant, pour reprendre l'expression souvent utilisée par Varela (p. ex. 1987; et empruntée au poète Antonio Machado). Nous espérons ainsi répondre en quelque sorte à l'appel de Pimm (2006), qui souligne l'urgence de concevoir des solutions de rechange à l'intemporalisation, à l'impersonnalisation et à la décontextualisation des mathématiques par lesquelles on se tient éloigné de la vie et de ceux qui font (des) mathématique(s). Nous avons à quelques reprises évoqué l'aspect créateur de l'activité mathématique vu sous cet angle. Une autre idée-force que l'on peut dégager d'une telle approche est celle du risque associé à toute activité mathématique. Problématiser, c'est créer une ouverture dans laquelle on s'avance sans une idée claire de ce qui nous attend, c'est s'ouvrir à l'invisible, à l'inconnu (p. ex. Roth, 2012). C'est, comme dirait Derrida (1987), invenir: à la fois créer et laisser venir une altérité hétérogène et incalculable, préparer sa venue, prendre le risque, la chance de lui ouvrir le passage, alors qu'au moment même de problématiser aucun calcul ne permet de prévoir où cette activité mathématique nous conduira. L'accent est alors véritablement mis sur l'action mathématique, cette pensée disait Bachelard où «l'imprudence est une méthode».

En parlant d'une nouvelle approche de l'activité mathématique (et) de l'élève, ce qui nous guide n'est pas l'idée d'un rapprochement au sens géométrique du terme, soit une réduction de la distance entre un point de départ et un point d'arrivée. C'est plutôt l'image du rapprochement qui vient en cheminant avec quelqu'un, avançant l'un avec l'autre. L'image d'une rencontre où les détours et les embûches deviennent un monde en commun. Trouvera-t-on dans cette approche une réponse à l'appel de Silver (2013), qui souligne le besoin urgent de développements théoriques permettant d'expliquer le lien entre résoudre et (com)poser des problèmes? Peut-être...

Tout passe et tout demeure Mais notre affaire est de passer De passer en traçant Des chemins Des chemins sur la mer A. Machado (1973, p. 209) 


\section{Références bibliographiques}

ARMSTRONG. A. C. (2013). Problem Posing as Storyline: Collective Authoring of Mathematics by Small Groups of Middle School Students. Thèse doctorale, University of British Columbia, Vancouver, C.-B.

BACCHI, C. (2012). Why study problematizations? Making politics visible. Open Journal of Political Science, 2(1), 1-8.

BACHELARD, G. (1938). La formation de l'esprit scientifique. Paris, J. Vrin.

BANWELL, C. S., SAUNDERS, K. D. et TAHTA, D. S. (1972). Starting Points. Stradbroke: Tarquin.

BAUERSFELD, H. (1994). Réflexions sur la formation des maîtres et sur l'enseignement des mathématiques au primaire. Revue des sciences de l'éducation, 20(1), 175-198.

BLUM, W. et LEISS, D. (2005). How do students and teachers deal with mathematical modelling problems? Dans C. Haines, P. Galbraith, W. Blum et S. Khan (dir.), Mathematical Modeling (ICTMA-12). Education, Engineering and Economics (p. 222-231). Chichester: Horwood.

BROWN, L. et COLES, A. (2012). Developing «deliberate analysis» for learning mathematics and for mathematics teacher education. How the enactive approach to cognition frames reflection. Educational Studies in Mathematics, 80(1-2), 217-231.

BROUSSEAU, G. (1998). Théorie des situations didactiques. Grenoble: La pensée sauvage.

BROUSSEAU, G. (2005). Situations fondamentales et processus génétiques de la statistique. Dans A. Mercier et C. Margolinas (dir.), Balises en didactique des mathématiques. Cours de la $12^{\mathrm{e}}$ École d'été de didactique des mathématiques (p. 165-249). Grenoble: La pensée sauvage.

BROWN, S. I. et WALTER, M. I. (2005). The Art of Problem Posing ( $3^{\mathrm{e}}$ éd.). New York: Routledge.

CHRISTOU, C., MOUSOULIDES, N., PITTALIS, M., PANTAZI-PITTA, D. et SRIRAMAN, B. (2005). An empirical taxonomy of problem posing processes. ZDM, 37(3), 149-158.

DAVIS, B. et SIMMT, E. (2004). «Mathématiques pour l'enseignement»: Une recherche longitudinale sur les connaissances mathématiques des enseignants (ou celles dont ils auraient besoin). Actes du colloque 2004 du Groupe des didacticiens de mathématiques du Québec (p. 5-21). Québec: GDM.

DAVIS, B., SUMARA, D. et KIEREN, T. (1996). Cognition, co-emergence, curriculum. Journal of Curriculum Studies, 28(2), 151-169. 
DEACON, R. (2000). Theory as practice. Foucault's concept of problematization. Telos, 118, 127-142.

DELEUZE, G. (1968). Différence et répétition. Paris: Presses universitaires de France.

DERRIDA, J. (1987). Psyché. Inventions de l'autre (tome 1). Paris: Galilée.

DUNCKER, K. (1945). On problem-solving. Psychological Monographs, 58(5).

Educational Studies in Mathematics (2013). Dossier thématique. PME Special Issue: Problem Posing in Mathematics Teaching and Learning: Establishing a Framework for Research, 83(1).

FABRE, M. (2009). Philosophie et pédagogie du problème. Paris: Vrin.

FOUCAULT, M. (1985). Michel Foucault, une histoire de la vérité. Paris: Syros.

GOUVERNEMENT DU QUÉBEC (1988). Fascicule K. Guide pédagogique, primaire, mathématiques. Résolution de problèmes. Orientation générale. Québec: Ministère de l’Éducation.

HADAMARD, J. (1959). Essai sur la psychologie de l'invention dans le domaine mathématique. Paris: Librairie scientifique Albert Blanchard.

HERSHKOWITZ, R., SCHWARZ, B. et DREYFUS, T. (2001). Abstraction in context. Epistemic actions. Journal for Research in Mathematics Education, 32(2), 195-222.

KIEREN, T. E. (juin 1995). Teaching Mathematics (in-the-middle): Enactivist View on Learning and Teaching Mathematics. Paper presented at the Queens/Gage Canadian National Mathematics Leadership Conference, Queens University, Kingston, Canada.

KILPATRICK, J. (1987). Problem formulating. Where do good problems come from? Dans A. H. Schoenfeld (dir.), Cognitive Science and Mathematics Education (p. 123-147). Hillsdale, NJ: Erlbaum.

LEWIS, T., PETRINA, S. et HILL, A. M. (1998). Problem posing - adding a creative increment to technological problem solving. Journal of Industrial Teacher Education, 36(1).

MACHADO, A. (1973). Champs de Castille. Paris : Gallimard.

MAHEUX, J.-F. et PROULX, J. (2014). Vers le faire mathématique. Essai pour un nouveau positionnement en didactique des mathématiques. Annales de didactique et de sciences cognitives, 19.

MATURANA, H. R. (1987). Everything is said by an observer. Dans W. I. Thompson (dir.), Gaia: A way of knowing (p. 65-82). Hudson, NY: Lindisfarne Press.

MATURANA, H. R. (1988). Reality: The search for objectivity of the quest for a compelling argument. Irish Journal of Psychology, 9(1), 25-82.

MATURANA, H. R. et VARELA, F. J. (1992). The Tree of Knowledge: The Biological Roots of Human Understanding (éd. rev.). Boston, MA: Shambhala. 
MATURANA, H. R. et VARELA, F. J. (1994). L'arbre de la connaissance. Paris: Addison-Wesley France.

MEYER, M. (1986). De la problématologie: philosophie, science et langage. Bruxelles: P. Mardaga.

PIMM, D. (2006). Drawing on the image in mathematics and art. Dans N. Sinclair, D. Pimm, W. Higginson (dir.), Mathematics and the Aesthetic. New Approaches to an Ancient Affinity (p. 160-190). Berlin: Springer.

POIRIER, L. (2000). Enseigner les maths au primaire, notes didactiques. Montréal: Renouveau pédagogique.

POLYA, G. (1965). Mathematical Discovery: On Understanding, Learning and Teaching Problem Solving (vol. II). New York: John Wiley \& Sons.

PONTE, J. et MATOS, J. F. (1992). Cognitive processes and social interactions in investigation activities. Dans J. Ponte et al. (dir.), Mathematical Problem Solving and New Information Technologies: Research in Contexts of Practice (p. 239-254). Berlin: Springer.

PROULX, J. (2010). Les objectifs et les illusions de la recherche en didactique des mathématiques. Réflexions et parcours de jeune chercheur. Dans J. Proulx (dir.), Actes de la journée d'étude sur l'entrée du jeune chercheur dans le milieu de la recherche en didactique des mathématiques: aléas, expériences et significations (p. 43-50). Montréal.

PROULX, J. (2013a). Le calcul mental au-delà des nombres: conceptualisations et illustrations avec la résolution d'équations algébriques. Annales de didactique et de sciences cognitives, 18, 61-90.

PROULX, J. (2013b). Mental mathematics, emergence of strategies, and the enactivist theory of cognition. Educational Studies in Mathematics, 84(3), 309-328.

PROULX, J. (2014). Mental mathematics and operations on functions. Proceedings of the Joint Meeting of the 38th Conference of the International Group for the Psychology of Mathematics Education and the $36^{\text {th }}$ Conference of Psychology of Mathematics Education, North American Chapter (vol. 5, p. 17-24). Vancouver, Canada.

PROULX, J., SIMMT, E. et TOWERS, J. (2009). RF05: The enactivist theory of cognition and mathematics education research. Issues of the past, current questions and future directions. Dans M. Tzekaki, M. Kaldrimidou et H. Sakonidis (dir.), Proceedings of the $33^{\text {rd }}$ Conference of the International Group for the Psychology of Mathematics Education (vol. 1, p. 249-278). Thessalonique, Grèce.

RADFORD, L. (1996). La résolution de problèmes: comprendre puis résoudre? Bulletin de l'Association mathématique du Québec (AMQ), 36(3), 19-30. 
REID, D. (1996). Enactivism as a methodology. Dans L. Puig et A. Gutiérrez (dir.), Proceedings of the Twentieth Annual Conference of the International Group for the Psychology of Mathematics Education (vol. 4, p. 203-210). Valence, Espagne: PME.

RENÉ DE COTRET, S. (1999). Perspective bio-cognitive pour l'étude des relations didactiques. Dans G. Lemoyne et F. Conne (dir.), Le cognitif en didactique des mathématiques (Cognition in Mathematics Education) (p. 103-120). Montréal: Les Presses de l'Université de Montréal.

REID, D., BROWN, L., COLES, A. et LOZANO, L. (à paraître). ZDM, numéro spécial Enactivist Methodology in Mathematics Education Research.

ROGALSKI, J. (2003). Y a-t-il un pilote dans la classe? Une analyse de l'activité de l'enseignant comme gestion d'un environnement dynamique ouvert. Recherches en didactique des mathématiques, 23(3), 343-388.

ROTH, W. M. (2012). Mathematical learning, the unseen and the unforseen. For the Learning of Mathematics, 32(3), 15-21.

SEVIM, V. et CIFARELLI, V. V. (2013). The co-evolution of problem posing and problem solving in the course of Sarah's on-going solution activity. Dans A. M. Lindmeier et A. Heinze (dir.), Proceedings of the $37^{\text {th }}$ Conference of the International Group for the Pyschology of Mathematics Education, 2, 137-144. Kiel, Allemagne: PME.

SILVER, E. A. (1994). On mathematical problem posing. For the Learning of Mathematics, 14(1), 19-28.

SILVER, E. A. (2013). Problem-posing research in mathematics education. Looking back, looking around, and looking ahead. Educational Studies in Mathematics, 83(1),157-162.

SIMON, R. (1985). A frog's eye view of the world. Structure is destiny: An interview with Humberto Maturana. The Family Therapy Networker, 9(3), 32-37 et 41-43.

SIMMT, E. (2000). Mathematics Knowing in Action: A Fully Embodied Interpretation. Thèse de doctorat, University of Alberta, Edmonton, Canada.

THOM, J. S., NAMUKASA, I. K., IBRAHIM-DIDI, K. et MCGARVEY, L. M. (2009). Perceptually guided action. Invoking knowing as enaction. Dans M. Tzekaki, M. Kaldrimidou et H. Sakonidis (dir.), Proceedings of the $33^{\text {rd }}$ Conference of the International Group for the Psychology of Mathematics Education (vol. 1, p. 252-256). Thessalonique, Grèce: PME.

VARELA, F. J. (1987). Laying down a path in walking. Dans W. I. Thompson (dir.), Gaia: A Way of Knowing. Political Implications of the New Biology (p. 48-64). New York: Lindisfarne Press.

VARELA, F. J. (1988). Le cercle créatif. Dans P. Watzlawick (dir.), L'invention de la réalité. Contributions au constructivisme (p. 329-345). Paris: Seuil. 
VARELA, F. J. (1996/1988). Invitation aux sciences cognitives (P. Lavoie, trad.). Paris: Seuil.

VARELA, F. J. (1999). Ethical Know-How: Action, Wisdom, and Cognition. Stanford: Stanford University Press.

VARELA, F. J., THOMPSON, E. et ROSCH, E. (1991). The Embodied Mind. Cognitive Science and Human Experience. Cambridge, MA: MIT Press.

VERA, D. et CROSSAN, M. M. (2004). Theatrical improvisation. Lessons for organizations. Organization Studies, 25(5), 727-749.

VOICA, C., PELCZER, I., SINGER, F. M. et CULLEN, C. (2013). When (and how) does a posed problem become a problem? Dans A. M. Lindmeier et A. Heinze (dir.), Proceedings of the $37^{\text {th }}$ Conference of the International Group for the Pyschology of Mathematics Education (vol. 2, p. 137-144). Kiel, Allemagne: PME.

WHITIN, D. J. (2004). Building a mathematical community through problem posing. Dans R. N. P. Rubenstein et G. W. Bright (dir.), Perspectives on the Teaching of Mathematics. Sixty-sixth Yearbook (p. 129-140). Reston, VA: National Council of Teachers of Mathematics.

XIN, Y. P. (dir.) (2012). Conceptual Model-Based Problem Solving. Taipei: Sense Publishers.

XIN, Y. P. et ZHANG, D. (2011). Exploring a conceptual model-based approach to teaching situated word problems. A rejoinder to Jitendra's response to Yan Ping Xin and Dake Zhang. The Journal of Educational Research, 104(5), 375-379.

ZACK, V. et REID, D. A. (2003). Good-enough understanding. Theorising about the learning of complex ideas (part 1), For the Learning of Mathematics, 23(3), 43-50.

ZACK, V. et REID, D. A. (2004). Good-enough understanding. Theorising about the learning of complex ideas (part 2), For the Learning of Mathematics, 24(1), 24-28. 